\title{
Thermal Gradient Mechanical Fatigue Assessment of a Nickel-Based Superalloy
}

\author{
Huang Yuan ${ }^{l}$, Jingyu Sun \\ Tsinghua University, School of Aerospace Engineering, Beijing 100084, China
}

\begin{abstract}
Turbine components generally work under thermomechanical loading conditions with varying temperature and significant temperature gradients. In the present work, a radiation heating system was developed to simulate thermal gradient mechanical fatigue (TGMF) loads in turbines. The specimen is externally heated by radiation and internally cooled by compressed air. Experiments showed that the TGMF life of the nickel-based superalloy is significantly shorter than that of the thermomechanical and the isothermal fatigue, although the thermal stress amplitude related to the temperature gradient is small. It was confirmed that the conventional fatigue models generated seriously deviations and could not catch effects of thermal gradients. The modified TGMF model introduced a correction term of the temperature gradient effects and can describe the TGMF lifetime of Inconel 718 reasonably. The new model provides a uniform description of isothermal and complex thermomechanical fatigue.
\end{abstract}

\section{INTRODUCTION}

The nickel-base superalloy Inconel 718 is widely used in the gas turbine engines [1]. In the past decades, isothermal fatigue (IF) tests were commonly used to predict the lifetime of gas turbine components, which generally work under varying thermomechanical loading conditions. Numerous investigations on the elevated temperature LCF of the nickel-based superalloy were performed [2-4]. Recent studies showed that the thermomechanical fatigue (TMF) loads influenced the fatigue damage mechanisms significantly [5-9] and the TMF lifetime can be significantly shorter than the isothermal one [10]. The turbine parts suffer from thermomechanical fatigue damage caused by the varying loads and temperatures. The temperature gradient brings the multiaxial stress state into the mechanical parts and makes the fatigue multiaxial. However, few works on the TGMF fatigue were published.

Brendel et al. [11] and Prasad et al. [12] investigated temperature gradients in TMF specimens. The results showed that the temperature gradients had a remarkable influence on the TMF lifetime. Baufeld et al. [13] and Bartsch et al. [14] carried out the thermal gradient mechanical fatigue testing for the single crystal superalloy CMSX-4. Microstructural changes, defects, phase evolutions of the metal coatings and rafting of the $\gamma / \gamma^{\prime}$ substrate morphology were investigated. They detected the cracks at the inner specimen surface and substrate pores. However, the fatigue life of the TGMF was not estimated. To quantify effects of temperature

\footnotetext{
${ }^{1}$ Corresponding author: yuan.huang@tsinghua.edu.cn
} 
gradients in thermomechanical fatigue and to establish a uniform fatigue life prediction model for complex thermomechanical fatigue, detailed TGMF tests are necessary, in combining with the investigation of constitutive modeling.

The induction heating has the advantage of the high heating rate as well as the high efficiency and is widely used in TMF testing $[9,11,15,16]$. Due to the skin effect of the induced current, however, the induction heating only heats the surfaces of the specimen, and the higher the induction frequency, the shallower the heating depth. For thin-walled specimens with a thickness of ca. $1 \mathrm{~mm}$, the inner and outer surfaces of the specimen are heated simultaneously, which makes the temperature distribution homogeneous in the thickness and does not meet the temperature distribution in real working condition [11].

In the present work, a radiation heating system is developed based on extensive finite element computations [17]. The numerical results are verified by experimental measurements and provide the basis for TGMF tests. LCF behavior of the nickel-based superalloy is experimentally tested under given both thermomechanical and thermal gradient mechanical loading conditions. The systematic experimental results provide a database for assessing fatigue life models and for establishing new TGMF fatigue life concepts.

\section{Experiments and results}

\subsection{Materials and specimens}

The nickel-based superalloy Inconel 718 investigated in the present paper was provided by ThyssenKrupp VDM GmbH. Fatigue testing specimens were in the thin-walled tubular shape with overall length of $120 \mathrm{~mm}$, a gauge length of $30 \mathrm{~mm}$, an outer diameter of the gauge section of $8.5 \mathrm{~mm}$ and an inner diameter of $6.5 \mathrm{~mm}$. The wall thickness of the tubular specimen in the gauge section is $1 \mathrm{~mm}$. All specimens were manufactured in a CNC machining center, and the surfaces were polished.

The thermal gradient mechanical fatigue (TGMF) testing system for the thin-walled tubular specimen was developed, as shown in Fig. 1 [17]. The specimen in the TGMF tests were heated by focused radiation heating from quartz lamps. Radiant heating can heat metals, non-metals, composites, etc., and can obtain complex temperature fields through multi-temperature zone control. The total power of the radiation furnace is $6.4 \mathrm{~kW}$ and can heat the tubular specimens to melt within seconds. The system includes multiple subsystems such as loading, heating, air cooling, and water cooling. These subsystems work cooperatively to ensure the mechanical load and temperature over the specimen gauge section are simultaneously varied and independently controlled.

The two air compressors were used to provide the cooling air of the specimen

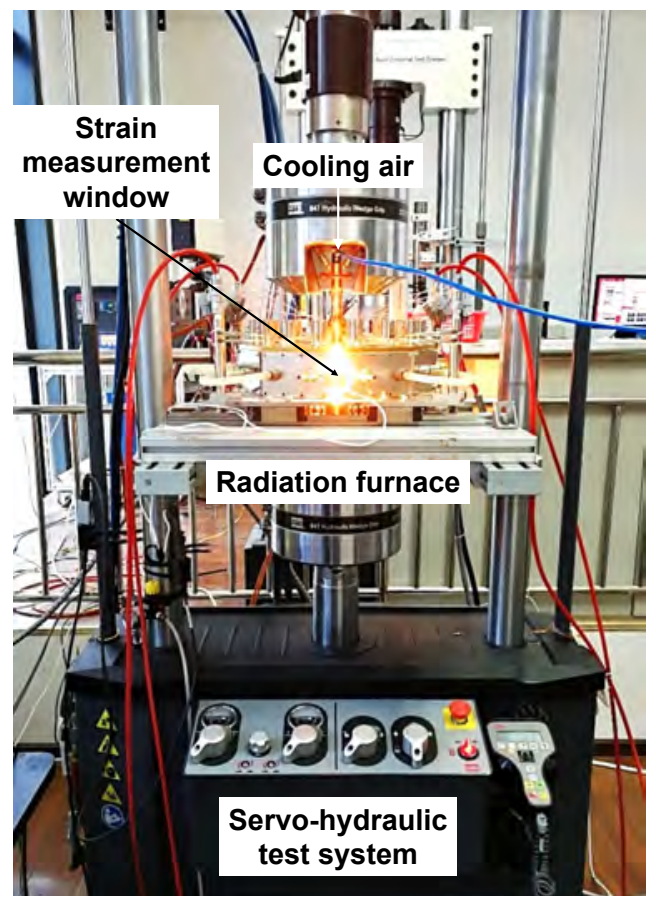

Fig. 1. Experimental apparatus of TGMF test system. 
and the lamp holders, separately. During all of the TGMF tests, the pressure and the flow volume of the compressed air were kept as constant to $40 \mathrm{l} / \mathrm{min}$. The metering valve was used to control the volume flow of the compressed air. The air-cooling subsystem also provides compressed air for the lamp holders. The duration of the TGMF test is usually long, so the lamp holders have to be cooled to ensure that they can work properly.

\subsection{Results of TGMF tests}

In the present work both isothermal and thermomechanical fatigue tests were run. The isothermal fatigue (IF) tests were performed at $650^{\circ} \mathrm{C}$, and the in-phase thermomechanical fatigue (TMF-IP) and the out-of-phase thermomechanical fatigue (TMF-OP) tests were carried out with a temperature range of $300 \sim 650^{\circ} \mathrm{C}$, as introduced in $[10,16]$. In order to compare with the TMF testing results, the TGMF tests with the same temperature range of $300 \sim 650^{\circ} \mathrm{C}$ and two kinds of phase angles were carried out: $0^{\circ}$ (IP) and $180^{\circ}$ (OP). Each kind of TGMF tests was conducted with four or five different mechanical strain amplitudes. The load type TGMF-IP stands for the in-phase thermal gradient mechanical fatigue, and TGMFOP means the out-of-phase thermal gradient mechanical fatigue. The nickel-based superalloy Inconel 718 used in $[10,16]$ was investigated in the present work under the TGMF loading conditions. Therefore, it is reasonable to compare the fatigue performance each other.

Because of the thermal gradients in radial and axial directions, the axial stress is not constant on the cross-section of the specimen. Therefore, the stress distribution of the specimen during the test has to be obtained with the help of finite element computations. In order to facilitate the comparison and analysis between the computational and experimental results, the nominal axial stress $\sigma$ on the cross-section defined as $\sigma=F / A$.

For fatigue tests under mechanical strain control, the fatigue life can generally be expressed as a function of mechanical strain amplitude. Fig. 2 shows the comparison of the fatigue lifetimes of TMF and TGMF tests under in-phase and out-of-phase loading conditions, respectively. The scattering of the experimental data is dramatic, although the isothermal fatigue life (IF) was obtained from the material at the upper temperature $650^{\circ} \mathrm{C}$. All the TMF and TGMF results show much lower fatigue lives than the IF. This result confirms that the fatigue life assessment based on the isothermal fatigue is generally not conservative for the Inconel 718. The TGMF-IP life is much smaller than the TMF-IP life, as shown in Fig. 2, and the TGMF-OP life is smaller than the TMF-OP life. The difference is substantial. In the figure the temperature cycles of the TMF and TGMF tests are the same, the only difference between the TMF and TGMF tests is the temperature gradient in the specimen. Therefore, the temperature gradient is considered to have a significant effect on the fatigue life under the thermomechanical loading condition.

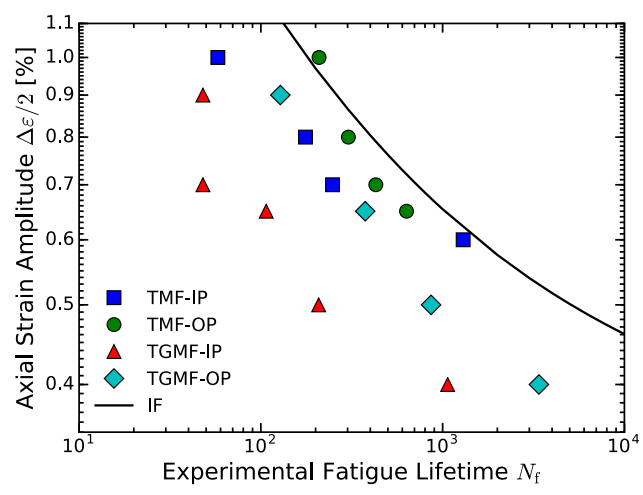

Fig. 2. Comparison of the fatigue lives under isothermal (IF), thermomechanical (TMF) and thermal gradient mechanical fatigue (TGMF) loading conditions. 
Figure 2 shows variation of the mean stress in the TGMF tests under IP and OP loading conditions. Since there is a phase difference between the temperature cycle and the load cycle in the fatigue test, the tensile half cycle and the compressive half cycle have different cycle softening behaviors. For the TGMF-IP test, an evident cyclic softening was observed during the tensile half cycle, whereas for the TGMF-OP test, the alloy performed a significant cyclic softening during the compressive half cycle. Fig. 3 illustrates the mean stress response

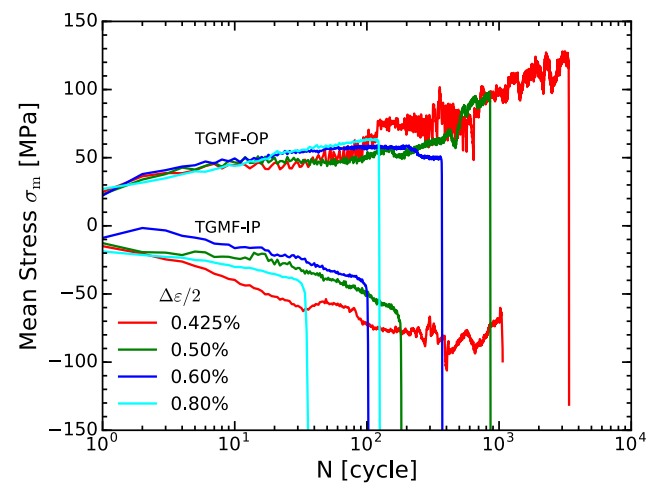

Fig. 3. Variations of the mean stress under TGMF IP and TGMF-OP loading conditions. curve for the Inconel 718 alloy under the in-phase and reverse phase TGMF test conditions. It can be seen that for TGMF-IP, the mean stress is compressive stress, and the increase of the mechanical strain amplitude has few effects on the mean stress. For TGMF-IP test, the mean stress becomes compression stress since the lower temperature stress is compressive. When $\Delta \varepsilon=0.8 \%$, the mean stress at the half-life cycle is about -30MPa. For TGMF-OP test, the mean stress becomes tensile and increases with the mechanical strain amplitude. When $\Delta \varepsilon=0.8 \%$, the mean stress at the halflife cycle is about $50 \mathrm{MPa}$. The asymmetry of cyclic softening in tension and compression results in variations of the mean stress in the fatigue tests towards the lower temperature loading direction. For different mechanical strain amplitudes, the mean stress evolution rate is similar, and the mean stress value increases with the number of cycles.

The evolution of mean stress in TGMF changes the strength of the alloy with temperature. Both in-phase and out-of-phase TGMF tests, the temperature cycles in the interval of 300 $\sim 650^{\circ} \mathrm{C}$. The material strength varies with temperature. When the temperature is high, the strength of the alloy is low. It results in the stress asymmetry of the hysteresis loop. The mean stress develops towards the low-temperature half cycle. The reason is that, with increasing mechanical strain amplitude, the plastic deformation of the alloy at the high temperature half cycle increases, which aggravates the asymmetry of tensile and compressive stress. Additionally, it can be observed that the absolute value of the mean stresses increases gradually with cycle under TGMF loading conditions, which can be considered as a result of the more intense softening of the superalloy Inconel 718 during the higher temperature half cycle. In the isothermal fatigue test, the mean stress value is generally small, so the mean stress has negligible variations on the isothermal fatigue test.

\section{Fatigue life assessment of TGMF}

\subsection{Fractography}

Analysis of fractography provides the locations of the crack initiation, the crack propagation and the direction of the fatigue striation. For both TGMF-IP and TGMF-OP loadings, crack initiation and subsequent failure have been identified as being initiated at the outer surface of the specimen [17]. Fig. 4 shows fractographies of the fatigue crack propagation. For the TGMF tests, the SEM investigations reveal that the dominant failure mechanism is changing with the phase angle of the thermal loading and mechanical loading, $\theta_{r \varepsilon}$. In Fig. 4(a) and (b) the fracture surfaces under TGMF-IP loading displays evident grain 
boundaries and slight fatigue striations. Consequently, it can be concluded that a mixture of transgranular and intergranular fracture mode is present in the TGMF-IP test, and the intergranular fracture plays a dominant role. Well-developed fatigue striations are observed in Figs. 3(c) and (d), which reveal transgranular crack growth is predominant during out-ofphase thermomechanical tests.

The present fractographic analysis confirms that the TGMF failure mechanisms are similar to the high temperature fatigue. All specimens show fatigue crack nucleation occurs in the outer specimen surfaces, which implies that the TGMF could be assessed by the conventional or TMF fatigue failure models. In the present section, several known fatigue models are selected to evaluate the fatigue life of the nickel-based superalloy under both TMF and TMGF loading conditions. Combined with the critical plane concept the models are well developed and verified for isothermal fatigue.
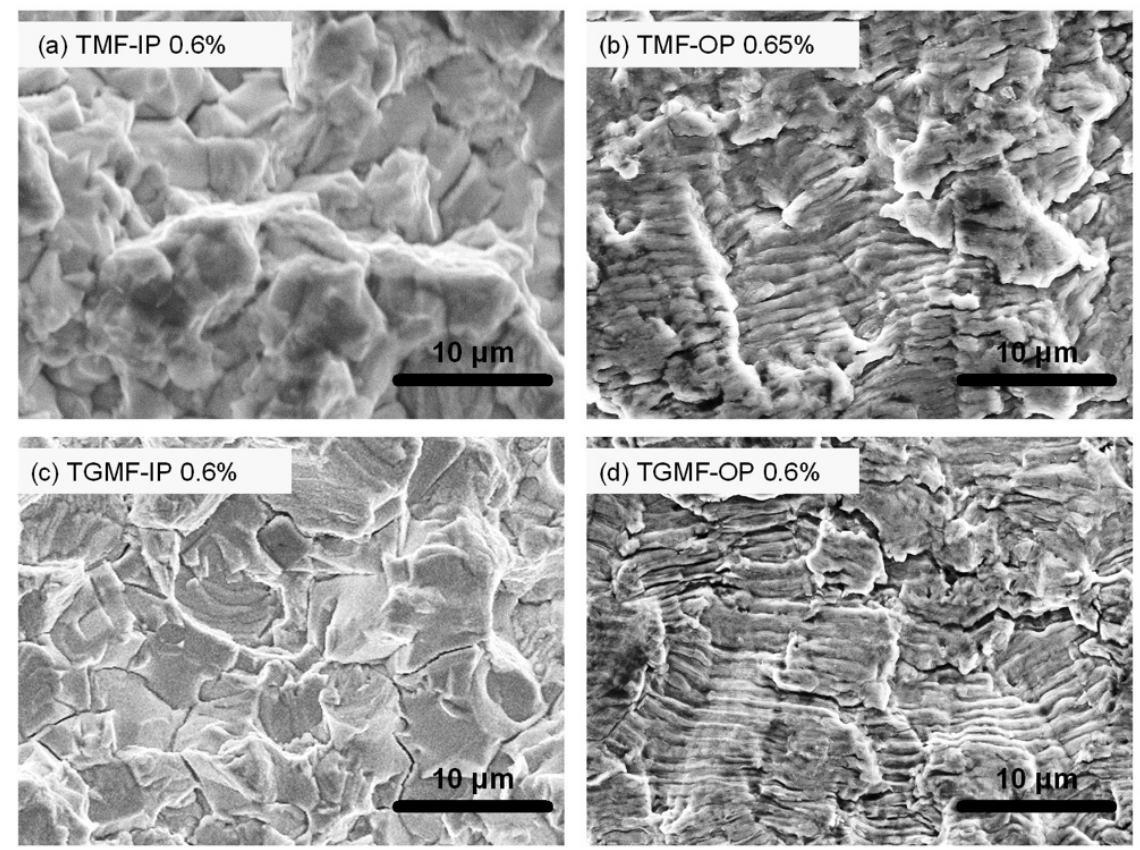

Fig. 4. Fractographs of fracture surfaces from the stable crack propagation areas of the TGMF specimens.

\subsection{Fatigue life prediction based on known models}

To verify the applicability of fatigue life models used to assess the fatigue life under both constant and varying temperature loading conditions, the following six models in combining with the critical plane concept are selected for the present TGMF cases: The Brown-Miller's model [18], Fatemi-Socie's model [19], Smith-Watson-Topper's model [20], Chu-ConleBonnen's model [21], Liu's Energy model [22] and the TMF model introduced by the authors [10]. Fig. 5 summarizes the comparison between predicted and experimental fatigue life of the IF, TMF-IP, TMF-OP, TGMF-IP, and TGMF-OP tests. In assessments, the computational stresses and strains are used to reduce effects of inhomogeneous stress and strain distributions induced by the temperature gradients.

As shown in the figure above, all the known models are not conservative and contain significant deviations in the mean life, besides the TMF and TGMF (To be introduced in the next section). The TMF model [10] gives the best prediction in the mean life prediction. The present model gives a reasonable mean life prediction with a small amount of conservative. 


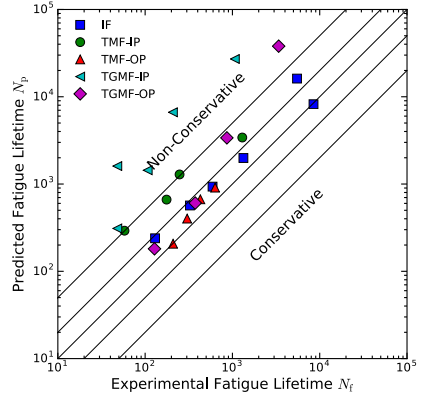

(a) Brown-Miller model

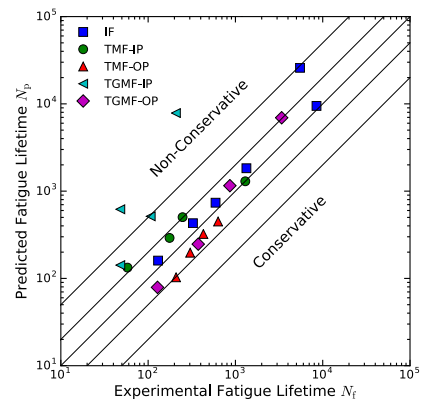

(d) Chu-Conle-Bonnen model

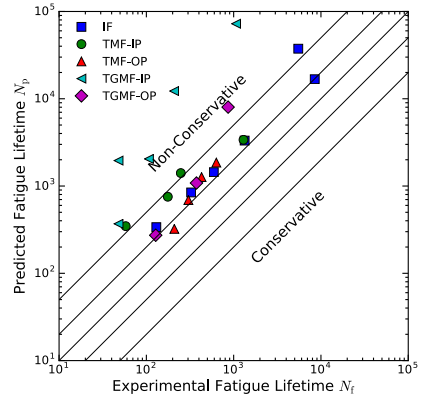

(b) Fatemi-Socie model

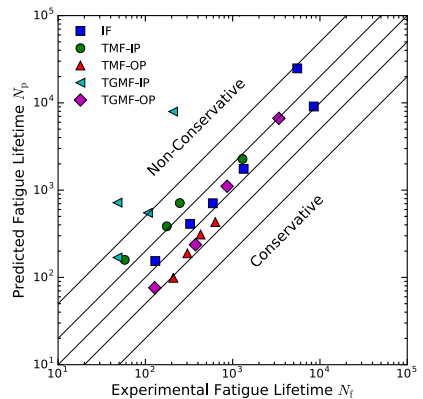

(e) Liu model

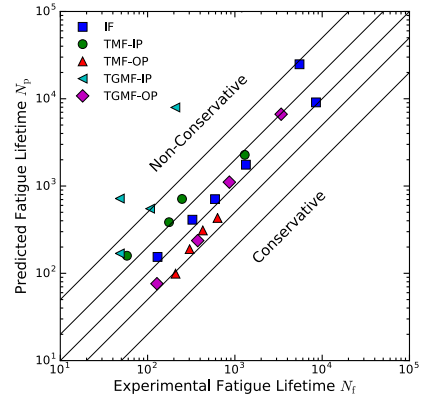

(c) Smith-Watson-Topper model

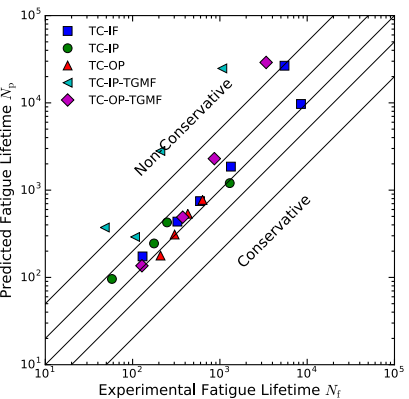

(f) TMF model

Fig. 5. Comparison between predicted fatigue life and experimental results of TMF as well as TGMF tests. (a) Brown-Miller model; (b) Fatemi-Socie model; (c) Smith-Watson-Topper model; (d) Chu-Conle-Bonnen model; (e) Liu Tension Energy model; (f) TMF model [10].

\subsection{Introduction of a new TGMF life model}

In the TGMF tests the thermal stress is induced by the temperature gradient and cannot be represented by the applied mechanical stress, which is the major difference to the TMF. To give a reasonable prediction of the TGMF life, the temperature gradient has to be included in the life model in a suitable way. The TMF model [10] seems to give the best agreement to both TMF and TGMF tests, but not conservative, and will be extended for TGMF in the present work.

Based on the TMF model [10], a correction term relying on the temperature gradient is to be included. The fatigue life model for TGMF is suggested as

$$
f(T, \nabla T)\left[A\left(\Delta \sigma_{n} \Delta \varepsilon_{n}\right)_{\max }+B(\Delta \tau \Delta \gamma)\right]\left(\frac{2}{1-R_{\sigma}}\right)=\frac{4 \sigma_{f}^{\prime 2}}{E}\left(2 N_{f}\right)^{2 b}+4{\sigma^{\prime}}_{f^{\prime}}{ }^{\prime}{ }_{f}\left(2 N_{f}\right)^{b+c} \text {. }
$$

The correction term $f$ depending on temperature and temperature gradient is introduced for correcting TGMF effects, as

$$
f(T, \nabla T)=1+\frac{g\|\nabla T\|}{T_{\text {melt }}-T_{\sigma_{n, \max }}},
$$

where $T_{\max }$ is the melting point of the material, and $T_{\sin m}$ is the temperature corresponding to the maximum normal stress, $g$ is a model parameter with the unit of $\mathrm{mm}$. For the Nickel-based superalloy Inconel $718, T_{\text {mil }}=1300^{\circ} \mathrm{C}$.

Figure 5 shows the fatigue life assessment results of the TGMF tests. Fig. 6(a) illustrates fatigue life curves for all fatigue tests and confirms the unique correlation between the fatigue damage parameter and life for both TMF and TGMF. Effects of temperature gradients are considered in the fatigue model properly. In Fig. 6(b), one can observe that deviations of the proposed model for most of the tests are within the scatter band with a factor of 2 . Only two 
tests reach the five-time scatter band. The present TGMF model generates the best mean value in comparing with all other models, while the present TGMF model possesses the least scattering. Combining the two parameters, the present TGMF model provides the best result in predicting the fatigue life for all experiments.
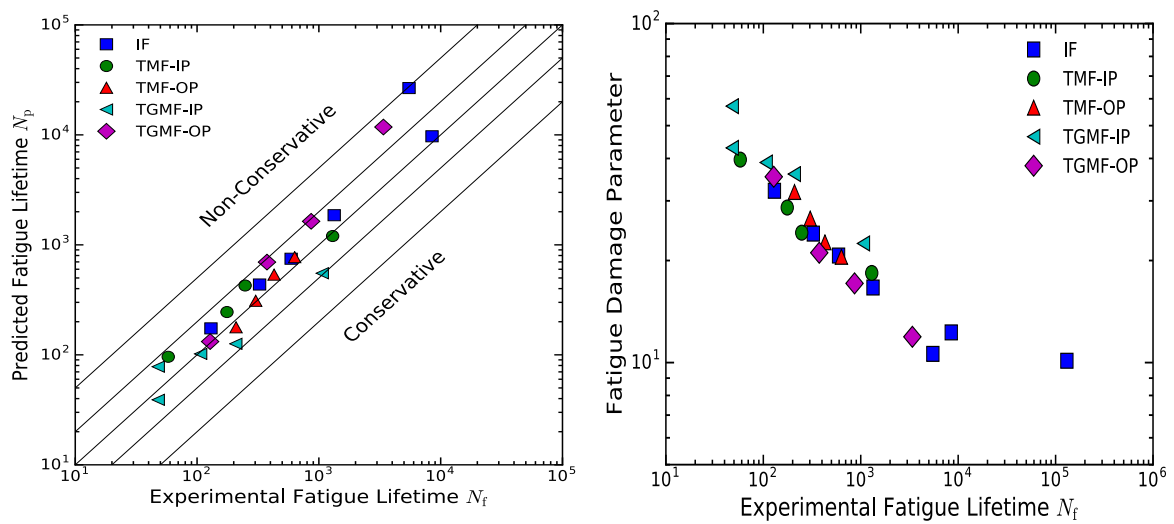

Fig. 6. Comparison of the TGMF tests, based on the present TGMF model. (a) Fatigue damage parameter versus experimental fatigue lifetime, (b) Comparison of the predicted fatigue lifetime and experimental fatigue lifetime.

\section{CONCLUSIONS}

The present paper experimentally investigated fatigue and its assessment for the nickelbased superalloy Inconel 718 under $300^{\circ} \mathrm{C} \sim 650^{\circ} \mathrm{C}$ thermomechanical and thermal gradient mechanical fatigue loading conditions. Effects of temperature gradients in combing with the phase angle are studied. The results can be extended to different temperature regions. The following conclusions can be drawn from the present work:

- Experimental results show that the temperature gradients change fatigue performance of the nickel-based alloy significantly and cannot be identified in thermomechanical fatigue. The phase angle affects the fatigue life assessment. The fractographic analysis reveals different failure mechanisms depending on TGMF loads.

- Conventional isothermal fatigue life models and TMF life models fail to account for TGMF influence and do not provide a satisfactory life prediction. Maximum deviations are larger than 10 times of the fatigue life. Effects of temperature gradients have to be considered in the fatigue model.

- Temperature gradients may introduce significant additional strains into the material. The mean stress in TMF tests varies with loading cycles and develops towards lower temperature loads. The variation of the mean stress makes it necessary to include characteristic features of the TGMF into the fatigue life model.

- A modified TGMF life model is proposed to consider influences from the temperature gradient, by introducing a correction term for the TMF loads. The modified TGMF model provides a significantly better agreement to the performed experiments and gives a uniform prediction for both TMF and TGMF fatigue.

- Further detailed TGMF experimental and computational investigations are necessary to understand the fatigue mechanisms, to quantify effects of temperature distributions and to establish a reliable life prediction model for mechanical parts under complex thermomechanical loading conditions. 


\section{References:}

1. Pollock, T.M and Tin, S. Nickel-Based Superalloys for Advanced Turbine Engines: Chemistry, Microstructure and Properties. Journal of Propulsion and Power, 22: 361374 (2006).

2 Koch, J.L. Proportional and non-proportional biaxial fatigue of Inconel 718. PhD thesis, University of Illinois, Department of Mechanical Engineering. 1985.

3 Mahobia, G.S., Paulose, N., Mannan, S.L., Sudhakar, R.G., Chattopadhyay, K., Santhi Srinivas, N.C., Singh, V. Effect of hot corrosion on low cycle fatigue behavior of superalloy IN718. International Journal of Fatigue, 59: 272-281 (2014).

4 G. Chen and Y. Zhang and D.K. Xu and Y.C. Lin and X. Chen. Low cycle fatigue and creep-fatigue interaction behavior of nickel-base superalloy GH4169 at elevated temperature of $650^{\circ} \mathrm{C}$. Materials Science and Engineering: A, 655: 175-182 (2016).

5 Evans, W. and Screech, J. and Williams, S. Thermo-mechanical fatigue and fracture of INCO718. International Journal of Fatigue, 30: 257-267 (2008).

6 Bauer, V. and Christ, H. J. Thermomechanical fatigue behaviour of a third generation $\gamma$ TiAl intermetallic alloy. Intermetallics, 17: 370-377 (2009).

7 D. Kulawinski and A. Weidner and S. Henkel and H. Biermann. Isothermal and thermomechanical fatigue behavior of the nickel base superalloy Waspaloy ${ }^{\mathrm{TM}}$ under uniaxial and biaxial-planar loading. International Journal of Fatigue, 81: 21-36 (2015).

8 M. Schlesinger and T. Seifert and J. Preussner. Experimental investigation of the time and temperature dependent growth of fatigue cracks in Inconel 718 and mechanism based lifetime prediction. International Journal of Fatigue, 99: 242 - 249 (2017).

9 W. Deng and J. Xu and Y. Hu and Z. Huang and L. Jiang. Isothermal and thermomechanical fatigue behavior of Inconel 718 superalloy. Materials Science and Engineering: A, 742: 813 - 819 (2019).

10 J. Sun and H. Yuan. Life assessment of multiaxial thermomechanical fatigue of a nickelbased superalloy Inconel 718. International Journal of Fatigue, 120: 228 - 240 (2019).

11 T. Brendel and E. Affeldt and J. Hammer and C. Rummel. Temperature gradients in TMF specimens. Measurement and influence on TMF life. International Journal of Fatigue, 2:234-240 (2008).

12 K. Prasad and V. Kumar. Temperature gradients in flat thermomechanical fatigue specimens. Applied Thermal Engineering, 59: 131 - 133 (2013)

13 B. Baufeld and M. Bartsch and M. Heinzelmann. Advanced thermal gradient mechanical fatigue testing of CMSX-4 with an oxidation protection coating, International Journal of Fatigue, 30: 219-225 (2008).

14 M. Bartsch and B. Baufeld and S. Dalkiliç and L. Chernova and M. Heinzelmann. Fatigue cracks in a thermal barrier coating system on a superalloy in multiaxial thermomechanical testing. International Journal of Fatigue, 30: 211 - 218 (2008).

15 J. Wang and Z. Moumni and W. Zhang. A thermomechanically coupled finite-strain constitutive model for cyclic pseudoelasticity of polycrystalline shape memory alloys. International Journal of Plasticity. 97: 194--221 (2017).

16 J. Sun and H. Yuan. Cyclic plasticity modeling of nickel-based superalloy Inconel 718 under multi-axial thermo-mechanical fatigue loading conditions. International Journal of Fatigue, 119: 89 - 101 (2019) 
17 J. Sun and H. Yuan. Thermal gradient mechanical fatigue assessment of a nickel-based superalloy. Submitted for publication (2019)

18 C.H. Wang and M.W. Brown. A path-independent parameter for fatigue under proportional and non-proportional loading. Fatigue and Fracture of Engineering Materials and Structures, 16:1285-1297 (1993).

19 A. Fatemi, D.F. Socie. A critical plane approach to multiaxial fatigue damage including out-of-phase loading. Fatigue and Fracture of Engineering Materials and Structures, 11:149-165 (1988).

20 K.N. Smith, P. Watson, T.H. Topper. A Stress-Strain Function for the Fatigue of Metals. Journal of Materials, 5:767-778 (1970).

21 C.C. Chu, F.A. Conle, J.J.F. Bonnen. Multiaxial stress-strain modeling and fatigue life prediction of SAE axle shafts. Advances in Multiaxial Fatigue, ASTM STP 1191, p. 37 54, 1993.

22 K.C. Liu. A method based on virtual strain-energy parameters for multiaxial fatigue life prediction. Advances in Multiaxial Fatigue, ASTM STP 1191, p. 67-84, 1993. 